\title{
Improved measurement of the reactor antineutrino flux at Daya Bay
}

D. Adey, ${ }^{1}$ F. P. An, ${ }^{2}$ A. B. Balantekin, ${ }^{3}$ H. R. Band, ${ }^{4}$ M. Bishai, ${ }^{5}$ S. Blyth,${ }^{6,7}$ D. Cao,${ }^{8}$ G. F. Cao, ${ }^{1}$ J. Cao, ${ }^{1}$ Y. L. Chan, ${ }^{9}$ J. F. Chang, ${ }^{1}$ Y. Chang, ${ }^{7}$ H. S. Chen, ${ }^{1}$ S. M. Chen, ${ }^{10}$ Y. Chen, ${ }^{11}$ Y. X. Chen, ${ }^{12}$ J. Cheng, ${ }^{13}$ Z. K. Cheng, ${ }^{14}$ J. J. Cherwinka, ${ }^{3}$ M. C. Chu, ${ }^{9}$ A. Chukanov,${ }^{15}$ J. P. Cummings,${ }^{16}$ F. S. Deng,${ }^{17}$ Y. Y. Ding, ${ }^{1}$ M. V. Diwan, ${ }^{5}$ M. Dolgareva,${ }^{15}$ J. Dove,${ }^{18}$ D. A. Dwyer, ${ }^{19}$ W. R. Edwards, ${ }^{19}$ M. Gonchar, ${ }^{15}$ G. H. Gong, ${ }^{10}$ H. Gong, ${ }^{10}$ W. Q. Gu, ${ }^{5}$ L. Guo ${ }^{10}$ X. H. Guo,${ }^{20}$ Y. H. Guo ${ }^{21}$ Z. Guo, ${ }^{10}$ R. W. Hackenburg, ${ }^{5}$ S. Hans,${ }^{5,}$ M. He, ${ }^{1}$ K. M. Heeger, ${ }^{4}$ Y. K. Heng, ${ }^{1}$ A. Higuera, ${ }^{22}$ Y. B. Hsiung, ${ }^{6}$ B. Z. Hu, ${ }^{6}$ T. Hu, ${ }^{1}$ Z. J. Hu, ${ }^{14}$ H. X. Huang, ${ }^{23}$ X. T. Huang, ${ }^{13}$ Y. B. Huang, ${ }^{1}$ P. Huber, ${ }^{24}$ W. Huo, ${ }^{17}$ G. Hussain, ${ }^{10}$ D. E. Jaffe, ${ }^{5}$ K. L. Jen, ${ }^{25}$ X. L. Ji, ${ }^{1}$ X. P. Ji, ${ }^{5}$ R. A. Johnson, ${ }^{26}$ D. Jones, ${ }^{27}$ L. Kang, ${ }^{28}$ S. H. Kettell, ${ }^{5}$ L. W. Koerner, ${ }^{22}$ S. Kohn, ${ }^{29}$ M. Kramer, ${ }^{19,29}$ T. J. Langford, ${ }^{4}$ L. Lebanowski, ${ }^{10}$ J. Lee ${ }^{19}$ J. H. C. Lee, ${ }^{30}$ R. T. Lei, ${ }^{28}$ R. Leitner, ${ }^{31}$ J. K. C. Leung, ${ }^{30}$ C. Li ${ }^{13}$ F. Li, ${ }^{1}$ H. L. Li, ${ }^{13}$ Q. J. Li ${ }^{1}$ S. Li ${ }^{28}$ S. C. Li, ${ }^{24}$ S. J. Li,${ }^{14}$ W. D. Li,${ }^{1}$ X. N. Li, ${ }^{1}$ X. Q. Li,${ }^{32}$ Y. F. Li, ${ }^{1}$ Z. B. Li, ${ }^{14}$ H. Liang, ${ }^{17}$ C. J. Lin, ${ }^{19}$ G. L. Lin, ${ }^{25}$ S. Lin, ${ }^{28}$ S. K. Lin, ${ }^{22}$ Y.-C. Lin, ${ }^{6}$ J. J. Ling, ${ }^{14}$ J. M. Link ${ }^{24}$ L. Littenberg, ${ }^{5}$ B. R. Littlejohn, ${ }^{33}$ J. C. Liu, ${ }^{1}$ J. L. Liu, ${ }^{34}$ Y. Liu, ${ }^{13}$ Y. H. Liu ${ }^{8}$ C. W. Loh, ${ }^{8}$ C. Lu, ${ }^{35}$ H. Q. Lu, ${ }^{1}$ J. S. Lu, ${ }^{1}$ K. B. Luk, ${ }^{29,19}$ X. B. Ma, ${ }^{12}$ X. Y. Ma, ${ }^{1}$ Y. Q. Ma, ${ }_{1}^{1}$ Y. Malyshkin, ${ }^{36}$ C. Marshall, ${ }^{19}$ D. A. Martinez Caicedo ${ }^{33}$ K. T. McDonald, ${ }^{35}$ R. D. McKeown, ${ }^{37,38}$ I. Mitchell, ${ }^{22}$ L. Mora Lepin, ${ }^{36}$ J. Napolitano, ${ }^{27}$ D. Naumov, ${ }^{15}$ E. Naumova, ${ }^{15}$ J. P. Ochoa-Ricoux,${ }^{36}$ A. Olshevskiy, ${ }^{15}$ H.-R. Pan, ${ }^{6}$ J. Park, ${ }^{24}$ S. Patton, ${ }^{19}$ V. Pec, ${ }^{31}$ J. C. Peng, ${ }^{18}$ L. Pinsky, ${ }^{22}$ C. S. J. Pun,${ }^{30}$ F. Z. Qi,${ }^{1}$ M. Qi ${ }^{8}$ X. Qian,,${ }^{5}$ R. M. Qiu, ${ }^{12}$ N. Raper,${ }^{14}$ J. Ren, ${ }^{23}$ R. Rosero, ${ }^{5}$ B. Roskovec ${ }^{36}$ X. C. Ruan, ${ }^{23}$ H. Steiner, ${ }^{29,19}$ J. L. Sun, ${ }^{39}$ K. Treskov, ${ }^{15}$ W.-H. Tse, ${ }^{9}$ C. E. Tull, ${ }^{19}$ B. Viren, ${ }^{5}$ V. Vorobel, ${ }^{31}$ C. H. Wang, ${ }_{1}^{7}$ J. Wang, ${ }_{14}^{14}$ M. Wang, ${ }^{13}$ N. Y. Wang,${ }_{5}^{20}$ R. G. Wang, ${ }^{1}$ W. Wang, ${ }^{14,38}$ W. Wang, ${ }^{8}$ X. Wang, ${ }^{40}$ Y. F. Wang, ${ }^{1}$ Z. Wang, ${ }^{1}$ Z. Wang, ${ }^{10}$ Z. M. Wang, ${ }^{1}$ H. Y. Wei, ${ }^{5}$ L. H. Wei, ${ }^{1}$ L. J. Wen, ${ }^{1}$ K. Whisnant, ${ }^{41}$ C. G. White, ${ }^{33}$ T. Wise, ${ }^{4}$ H. L. H. Wong, ${ }^{29,19}$ S. C. F. Wong, ${ }^{14}$ E. Worcester, ${ }^{5}$ Q. Wu, ${ }^{13}$ W. J. Wu, ${ }^{1}$ D. M. Xia, ${ }^{42}$ Z. Z. Xing, ${ }^{1}$ J. L. Xu, ${ }^{1}$ T. Xue, ${ }^{10}$ C. G. Yang, ${ }^{1}$ H. Yang, ${ }^{8}$ L. Yang, ${ }^{28}$ M. S. Yang, ${ }^{1}$ M. T. Yang, ${ }^{13}$ Y. Z. Yang, ${ }^{14}$ M. Ye, ${ }^{1}$ M. Yeh, ${ }^{5}$

B. L. Young, ${ }^{41}$ H. Z. Yu, ${ }^{14}$ Z. Y. Yu, ${ }^{1}$ B. B. Yue,${ }^{14}$ S. Zeng, ${ }^{1}$ L. Zhan, ${ }^{1}$ C. Zhang, ${ }^{5}$ C. C. Zhang, ${ }^{1}$ F. Y. Zhang, ${ }^{3}{ }^{\prime}$

H. H. Zhang, ${ }_{14}$ J. W. Zhang, ${ }^{1}$ Q. M. Zhang, ${ }^{21}$ R. Zhang, ${ }^{8}$ X. F. Zhang, ${ }^{1}$ X. T. Zhang, ${ }^{1}$ Y. M. Zhang, ${ }^{14}$ Y. M. Zhang, ${ }^{10}$ Y. X. Zhang, ${ }^{39}$ Y. Y. Zhang, ${ }^{34}$ Z. J. Zhang, ${ }^{28}$ Z. P. Zhang, ${ }^{17}$ Z. Y. Zhang, ${ }^{1}$ J. Zhao, ${ }^{1}$ P. Zheng, ${ }^{28}$

L. Zhou, ${ }^{1}$ H. L. Zhuang, ${ }^{1}$ and J. H. Zou ${ }^{1}$

(Daya Bay Collaboration)

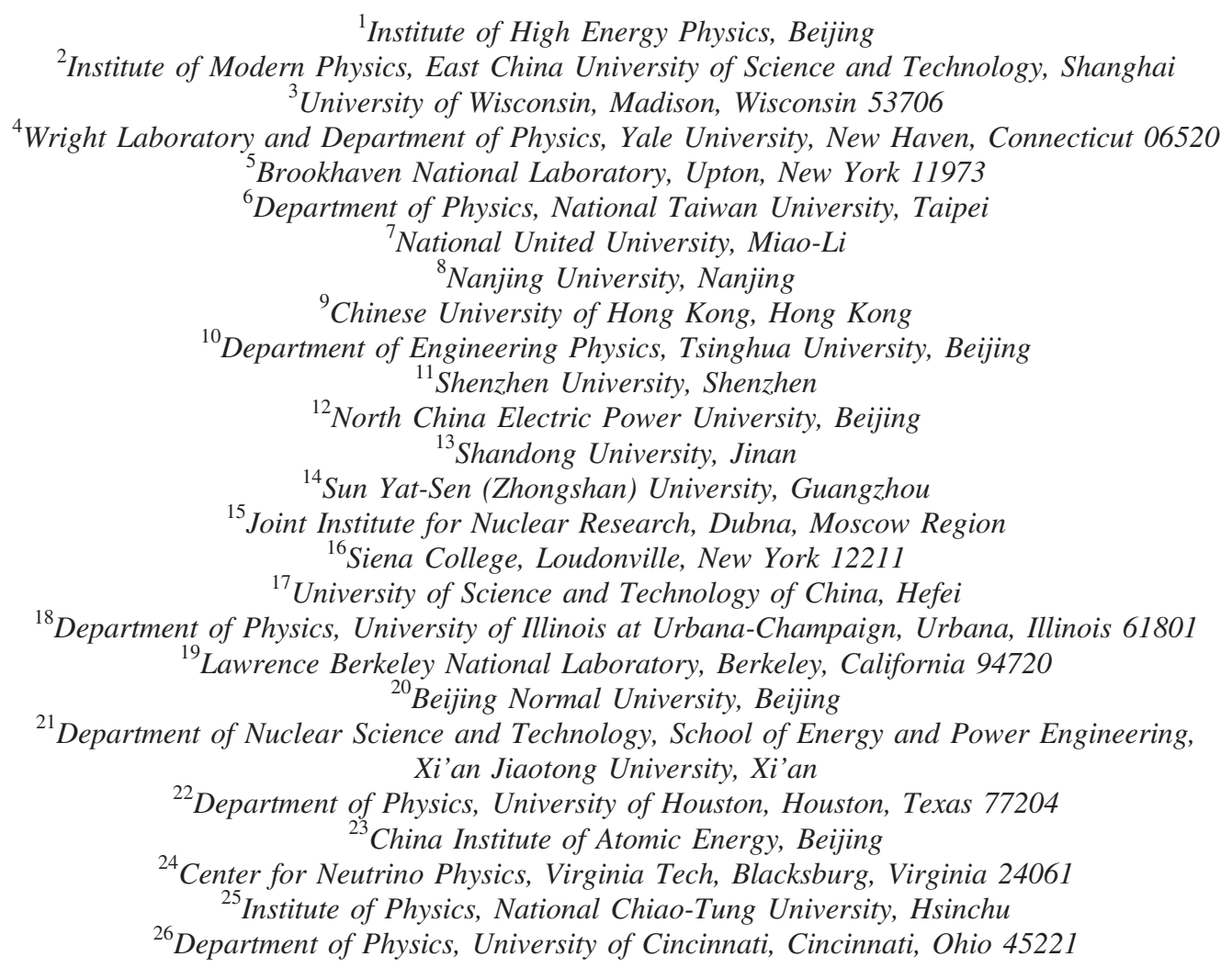




\author{
${ }^{27}$ Department of Physics, College of Science and Technology, Temple University, \\ Philadelphia, Pennsylvania 19122 \\ ${ }^{28}$ Dongguan University of Technology, Dongguan \\ ${ }^{29}$ Department of Physics, University of California, Berkeley, California 94720 \\ ${ }^{30}$ Department of Physics, The University of Hong Kong, Pokfulam, Hong Kong \\ ${ }^{31}$ Charles University, Faculty of Mathematics and Physics, Prague \\ ${ }^{32}$ School of Physics, Nankai University, Tianjin \\ ${ }^{33}$ Department of Physics, Illinois Institute of Technology, Chicago, Illinois 60616 \\ ${ }^{34}$ Department of Physics and Astronomy, Shanghai Jiao Tong University, Shanghai Laboratory for \\ Particle Physics and Cosmology, Shanghai \\ ${ }^{35}$ Joseph Henry Laboratories, Princeton University, Princeton, New Jersey 08544 \\ ${ }^{36}$ Instituto de Física, Pontificia Universidad Católica de Chile, Santiago \\ ${ }^{37}$ California Institute of Technology, Pasadena, California 91125 \\ ${ }^{38}$ College of William and Mary, Williamsburg, Virginia 23187 \\ ${ }^{39}$ China General Nuclear Power Group, Shenzhen \\ ${ }^{40}$ College of Electronic Science and Engineering, National University of Defense Technology, Changsha \\ ${ }^{41}$ Iowa State University, Ames, Iowa 50011 \\ ${ }^{42}$ Chongqing University, Chongqing
}

(Received 3 September 2018; published 9 September 2019)

\begin{abstract}
This work reports a precise measurement of the reactor antineutrino flux using 2.2 million inverse beta decay (IBD) events collected with the Daya Bay near detectors in 1230 days. The dominant uncertainty on the neutron detection efficiency is reduced by $56 \%$ with respect to the previous measurement through a comprehensive neutron calibration and detailed data and simulation analysis. The new average IBD yield is determined to be $(5.91 \pm 0.09) \times 10^{-43} \mathrm{~cm}^{2} /$ fission with total uncertainty improved by $29 \%$. The corresponding mean fission fractions from the four main fission isotopes ${ }^{235} \mathrm{U},{ }^{238} \mathrm{U},{ }^{239} \mathrm{Pu}$, and ${ }^{241} \mathrm{Pu}$ are $0.564,0.076,0.304$, and 0.056 , respectively. The ratio of measured to predicted antineutrino yield is found to be $0.952 \pm 0.014 \pm 0.023(1.001 \pm 0.015 \pm 0.027)$ for the Huber-Mueller (ILL-Vogel) model, where the first and second uncertainty are experimental and theoretical model uncertainty, respectively. This measurement confirms the discrepancy between the world average of reactor antineutrino flux and the Huber-Mueller model.
\end{abstract}

DOI: 10.1103/PhysRevD.100.052004

\section{INTRODUCTION}

Nuclear reactors are an intense manmade source of electron antineutrinos and were used for the first observation of the neutrino [1]. Electron antineutrinos can be detected through inverse beta decay (IBD) on target protons, where a prompt positron and a delayed neutron capture signals are measured in time coincidence. Since the early 2000s, the energy and baseline (the distance between source and detector) dependent neutrino disappearance at nuclear reactors [2-4] has provided strong evidence of neutrino oscillation [5-7]. However, a recent improved reevaluation of the theoretical prediction (referred to as Huber-Mueller model $[8,9]$ ) of the reactor neutrino flux

\footnotetext{
*Now at Department of Chemistry and Chemical Technology, Bronx Community College, Bronx, New York 10453, USA.

Published by the American Physical Society under the terms of the Creative Commons Attribution 4.0 International license. Further distribution of this work must maintain attribution to the author(s) and the published article's title, journal citation, and DOI. Funded by SCOAP .
}

resulted in a $\sim 6 \%$ deficit in measured flux from shortbaseline experiments [10] and the previous ILL-Vogel model [11-14]. The difference between the data and Huber-Mueller prediction, i.e., the so-called "reactor antineutrino anomaly" (RAA), could be interpreted as activeto-sterile neutrino oscillation with a mass-squared splitting $\left(\Delta m^{2}\right)$ around $1 \mathrm{eV}^{2}$. It is also shown in Refs. [15-17] that the allowed parameter space is compatible with earlier anomalies from LSND [18,19], MiniBooNE [20], GALLEX [21], and SAGE [22]. On the other hand, a number of authors [23-26] have argued that the RAA may be due to the theoretical uncertainties in the flux calculations. Recent antineutrino flux evolution results from Daya Bay are in tension with the sterile-neutrino-only explanation of RAA [27].

The uncertainty of the reactor antineutrino flux in our previous measurement [27] is dominated by the uncertainty of neutron detection efficiency. The neutron detection efficiency was determined to be $\varepsilon_{n}=(81.83 \pm 1.38) \%$ $[28,29]$, and the ratio with respect to the total uncertainty is $\sigma_{\varepsilon_{n}}^{2} / \sigma_{\text {total }}^{2}=65 \%$. To further elucidate the RAA situation, 
this work presents an updated flux measurement from Daya Bay using the same 1230-day data set, but with a more precise determination of the neutron detection efficiency. Key improvements include an elaborated neutron calibration campaign covering a wide range of neutron energy and positions, an improved simulation with different physics models, and a data-driven correction to the neutron efficiency.

This paper is organized as follows. In Sec. II, we explain the general method to measure reactor neutrino yield, and highlight our approach here to improve its estimate. Section III discusses the neutron calibration campaign and the analysis of calibration and simulation data. In Sec. IV we present an improved reactor antineutrino flux measurement and a comparison with the world data and theoretical models.

\section{METHOD}

\section{A. Overview of procedure}

The Daya Bay experiment has four near and four far identically designed antineutrino detectors (ADs), located at different baselines (360 m-1900 m) [30] measuring the electron antineutrino flux from six reactor cores. The structure of the detector is shown in Fig. 1. Each AD consists of a cylindrical target volume with 20 tons of $0.1 \%$ gadolinium $(\mathrm{Gd})$ loaded liquid scintillator (GdLS, $3.1 \mathrm{~m}$ in diameter and $3.1 \mathrm{~m}$ in height), surrounded by a layer of 42-cm thick liquid scintillator (LS) to enclose the gammas or electrons escaped from the central GdLS region. The GdLS and LS are separated by a $1-\mathrm{cm}$ thick acrylic vessel. An energy deposit in the GdLS and LS regions is detected by photomultiplier tubes (PMTs).

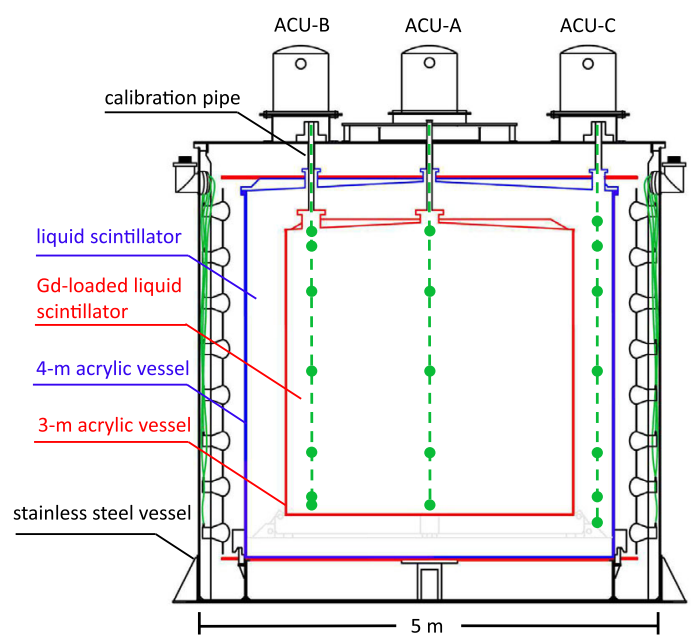

FIG. 1. Schematic diagram of the Daya Bay antineutrino detector. On the top, three automated calibration unit (ACU-A, $\mathrm{B}$, and $\mathrm{C}$ ), are installed. The three related vertical calibration axes are shown as the dashed lines, with all the locations of the calibration points highlighted.
The origin of the coordinate system is set at the geometrical center of the GdLS cylinder, with the $z$-axis pointing up. IBD neutrons are detected by delayed capture either on hydrogen emitting one $2.2 \mathrm{MeV}$ gamma or on gadolinium emitting several gammas with total energy of about $8 \mathrm{MeV}$. The kinetic energy of the IBD neutrons is less than $50 \mathrm{keV}$. The average capture time in the GdLS region is about $28.5 \mu \mathrm{s}$ and $216 \mu \mathrm{s}$ in the LS [31].

The reactor antineutrino IBD candidates are selected with the same criteria as in Ref. [32], which are also described here. (1) Removal of events caused by PMT light emission. (2) The time between the prompt and delayed signal is in the range of $[1,200] \mu \mathrm{s}$. (3) Prompt signal must have a reconstructed energy, E, between 0.7 and $12 \mathrm{MeV}$. (4) Delayed signal must have E between 6 and $12 \mathrm{MeV}$ to select gadolinium captures. (5) Muon anticoincidence. (6) Multiplicity cut to remove events with $\mathrm{E}>0.7 \mathrm{MeV}$ in the interval $200 \mu$ s before the prompt signal, $200 \mu$ s after the delayed signal, or between the prompt and delayed signals. The dominant backgrounds are accidental coincident events and cosmic-ray muon induced ${ }^{9} \mathrm{Li} /{ }^{8} \mathrm{He}$, which are less than $2 \%$ of the signal IBD rate for the four near ADs. After statistical subtraction of background, the total number of IBD signals, $N_{\mathrm{IBD}}$, is $2.201 \times 10^{6}$ for the four near detectors.

To compare to the theoretical predictions, the reactor antineutrino yield $\sigma_{f}$, defined as the number of antineutrinos times IBD cross section per fission, can be calculated by solving the following equation:

$$
N_{\mathrm{IBD}}\left(1-c^{\mathrm{SNF}}\right)=\sigma_{f} \sum_{d=1}^{4} \sum_{r=1}^{6} \frac{N_{d}^{P} \varepsilon_{\mathrm{IBD}} P_{\text {sur }}^{r d} N_{r}^{f}}{4 \pi L_{r d}^{2}},
$$

where the index $d$ is for four near detectors, index $r$ is for the six reactor cores, $N_{d}^{P}$ is the number of target protons of detector $d, \varepsilon_{\mathrm{IBD}}$ is the IBD detection efficiency, $P_{\text {sur }}^{r d}$ is the mean neutrino survival probability from the reactor $r$ to detector $d, N_{r}^{f}$ is the predicted number of fissions of the $r$ th reactor core, $L_{r d}$ is the distance from reactor $r$ to detector $d$, and $c^{\mathrm{SNF}}$ is a correction term for spent nuclear fuel. $P_{s u r}^{r d}$ is calculated by integrating the cross-sectionweighted oscillation survival probability over the $\bar{\nu}_{e}$ energy spectrum, using $\sin ^{2} 2 \theta_{13}$ and $\left|\Delta m_{e e}^{2}\right|$ determined from the same data [32]. The cross section is calculated using the formalism in [33], with the updated neutron lifetime in [34]. The average oscillation correction for near detectors is $1.5 \%$.

The IBD detection efficiency is divided into two factors:

$$
\varepsilon_{\mathrm{IBD}}=\varepsilon_{n} \times \varepsilon_{\mathrm{other}},
$$

where $\varepsilon_{n}$ is the neutron selection efficiency due to the $[6,12] \mathrm{MeV}$ cut and $\varepsilon_{\text {other }}$ is for the PMT light emission, prompt energy, and coincident time cuts. 
TABLE I. Summary of relative uncertainties for the flux measurements and the measured flux to model prediction ratio measurements in our previous study [29] and this work. Central values of the detector efficiencies, $\varepsilon_{n}$ and $\varepsilon_{\text {other }}$, are also listed.

\begin{tabular}{|c|c|c|c|c|}
\hline \multirow[b]{2}{*}{ Source } & \multicolumn{2}{|c|}{ Previous } & \multicolumn{2}{|c|}{ This work } \\
\hline & Value & Rel err & Value & Rel err \\
\hline Statistic & $\cdots$ & $0.1 \%$ & $\cdots$ & $0.1 \%$ \\
\hline Oscillation & $\cdots$ & $0.1 \%$ & $\cdots$ & $0.1 \%$ \\
\hline Target proton & $\cdots$ & $0.92 \%$ & $\cdots$ & $0.92 \%$ \\
\hline \multicolumn{5}{|l|}{ Reactor } \\
\hline Power & $\ldots$ & $0.5 \%$ & $\cdots$ & $0.5 \%$ \\
\hline energy/fission & $\ldots$ & $0.2 \%$ & $\ldots$ & $0.2 \%$ \\
\hline IBD cross section & $\ldots$ & $0.12 \%$ & $\ldots$ & $0.12 \%$ \\
\hline Fission fraction & $\cdots$ & $0.6 \%$ & $\cdots$ & $0.6 \%$ \\
\hline Spent fuel & $\ldots$ & $0.3 \%$ & $\ldots$ & $0.3 \%$ \\
\hline Nonequilibrium & $\ldots$ & $0.2 \%$ & $\ldots$ & $0.2 \%$ \\
\hline \multicolumn{5}{|l|}{$\varepsilon_{\mathrm{IBD}}$} \\
\hline$\varepsilon_{n}$ & $81.83 \%$ & $1.69 \%$ & $81.48 \%$ & $0.74 \%$ \\
\hline$\varepsilon_{\text {other }}$ & $98.49 \%$ & $0.16 \%$ & $98.49 \%$ & $0.16 \%$ \\
\hline Total & $\ldots$ & $2.1 \%$ & $\cdots$ & $1.5 \%$ \\
\hline
\end{tabular}

The predicted number of fissions of the $r$ th reactor core is

$$
N_{r}^{f}=\int \frac{W_{r}}{\sum_{\mathrm{iso}=1}^{4} f_{r}^{\text {iso }} E^{\text {iso }}} \mathrm{d} t,
$$

where $W_{r}$ is the thermal power of the $r$ th core, $E^{\text {iso }}$ is the mean energy released per fission for each isotope [35], and $f_{r}^{\text {iso }}$ is the average fission fraction of the $r$ th core for each isotope, and the ratio is integrated over the live time of the detectors. The original thermal power and fission fuel composition data are provided by the power plant. $c^{\mathrm{SNF}}$ was estimated to be $(0.3 \pm 0.3) \%$ previously [29].

Different components of relative uncertainties for the antineutrino yield measurement from previous work [28,29], including $\varepsilon_{n}$ and $\varepsilon_{\text {other }}$, are summarized in Table I. Clearly $\varepsilon_{n}$ dominates the uncertainty, and is the target of improvement in this paper.

\section{B. Principle of improvement}

The neutron detection efficiency, $\varepsilon_{n}$, is composed of three individual factors.

(i) The Gd capture fraction is the fraction of neutrons produced by IBD in the GdLS target that are captured on Gd. The capture fraction is lower at the edge of GdLS volume because neutrons may drift into undoped LS volume (spill-out effect).

(ii) The nGd gamma detection efficiency is the fraction of neutron Gd capture signals with detected gamma energy above $6 \mathrm{MeV}$.

(iii) Spill-in: efficiency increase due to IBD events produced in the LS and acrylic but with neutron capture on Gd.
We note that the estimation of Gd capture fraction and spill-in effects are strongly correlated since they are both driven by the modeling of neutron propagation including neutron scattering in materials and the subsequent nuclear capture. The estimation of the nGd gamma detection efficiency depends on the modeling of gamma emission including the multiplicity and energy spectrum of the emitted gammas.

In the previous study [29], we attempted to use different neutron calibration data to estimate these individual effects. The main difficulty was that no data can cleanly separate their uncertainties. In this paper, instead, we evaluate $\varepsilon_{n}$ and its uncertainty directly using a new neutron calibration data set and a data-simulation comparison. This approach is data driven and allows a significant reduction of the uncertainty.

\section{IMPROVED DETECTION EFFICIENCY ESTIMATION}

\section{A. Neutron calibration campaign}

An extensive neutron calibration campaign was carried out in Daya Bay at the end of 2016. Two types of custom sources were fabricated, ${ }^{241} \mathrm{Am}-{ }^{13} \mathrm{C}$ (AmC, neutron rate $\sim 100 \mathrm{~Hz}$ ) [36] and ${ }^{241} \mathrm{Am}-{ }^{9} \mathrm{Be}$ (AmBe, neutron rate $\sim 30 \mathrm{~Hz})$. They produce neutrons through ${ }^{13} \mathrm{C}(\alpha, \mathrm{n}){ }^{16} \mathrm{O}$ or ${ }^{9} \mathrm{Be}(\alpha, \mathrm{n}){ }^{12} \mathrm{C}$ reactions with the final nucleus either in the ground state (GS) or excited state (ES). The kinetic energy of the neutrons from $\mathrm{AmC}(\mathrm{AmBe})$ in the GS and $\mathrm{ES}$ are $[3,7] \mathrm{MeV}([6,10] \mathrm{MeV})$ and $<1 \mathrm{MeV}([2,6] \mathrm{MeV})$, respectively. Calibration events are formed from the prompt energy of the proton recoil and deexcitation gammas, if ${ }^{16} \mathrm{O}^{*}$ or ${ }^{12} \mathrm{C}^{*}$ is created, and the delayed neutron capture. The high neutron rate and delayed-time-coincidence present a high signal-to-background ratio for the calibration study.

The sources, sealed in a small stainless steel cylinder ( $8 \mathrm{~mm}$ in both diameter and height), were enclosed in a highly reflective PTFE (Polytetrafluoroethylene) shell. These sources were deployed vertically into a near-site $\mathrm{AD}$ using the automated calibration units (ACUs) [37] along the central axis (ACU-A), an edge axis of GdLS at a radius of $1.35 \mathrm{~m}$ (ACU-B), and through a middle axis of the LS layer at a radius of $1.77 \mathrm{~m}$ (ACU-C). During deployment, the absolute precision of the source $z$ location is $7 \mathrm{~mm}$ [37]. All calibration positions are illustrated in Fig. 1. In total, data in 59 different source (final nucleus states) and location points (SLPs), were collected.

Delayed coincidence events for the calibration sample are selected with a time requirement of $1 \mu \mathrm{s}<\Delta t<$ $1200 \mu \mathrm{s}$ for all events with $\mathrm{E}$ between $[0.3,12] \mathrm{MeV}$. The $1200 \mu$ s selection cut is set to efficiently include neutron captures in the LS and acrylic region. Two example distributions of the prompt-delay energies of AmC (ACU-B $z=0 \mathrm{~m}$ ) and $\mathrm{AmBe}(\mathrm{ACU}-\mathrm{B} z=0 \mathrm{~m}$ ) samples are shown 


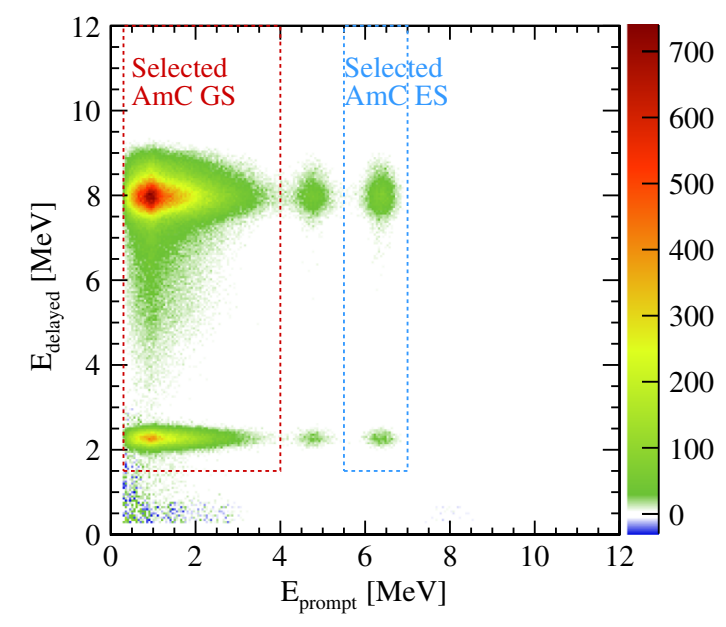

FIG. 2. The prompt vs delayed energy distribution of one AmC sample (ACU-B $z=0 \mathrm{~m}$ ). The selected ground state (GS) and excited state (ES) are indicated. The clusters in the prompt energy spectrum between $[4,5.5] \mathrm{MeV}$ are caused by ${ }^{12} \mathrm{C}(\mathrm{n}, \mathrm{n} \gamma){ }^{12} \mathrm{C}$, and are not used. Negative bin content is due to background subtraction.

in Figs. 2 and 3, respectively. The data in different channels are selected using the following prompt energy cut: $[0.3,4] \mathrm{MeV}$ for AmC GS, $[5.5,7] \mathrm{MeV}$ for AmC ES, $[1,4] \mathrm{MeV}$ for AmBe GS, and $[4.2,7] \mathrm{MeV}$ for AmBe ES. The accidental background contributes $0.1 \%-20 \%$ of the neutron candidates depending on the SLP, and is estimated by randomly paired single events [31]. The reactor antineutrino and cosmogenic backgrounds are estimated by applying the same selection cuts on the data acquired immediately before and after the calibration campaign. They contribute $<0.1 \%$ to the neutron source signals. All of these backgrounds are statistically subtracted.

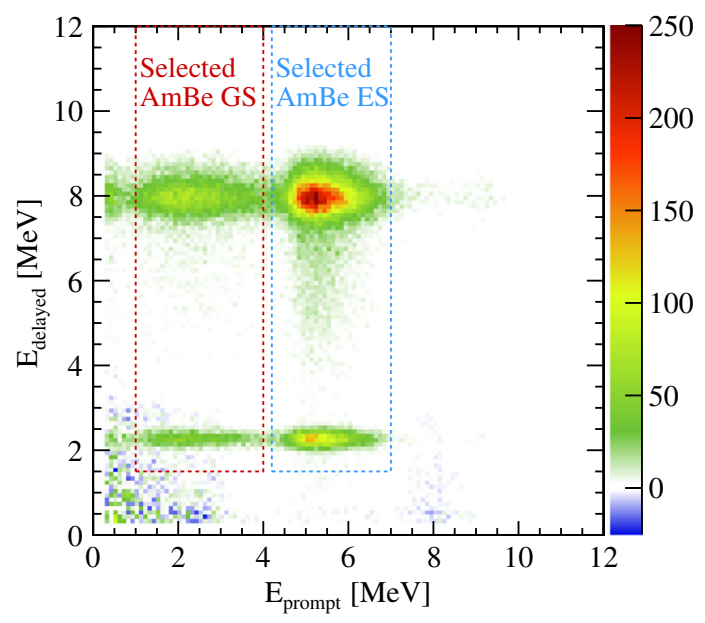

FIG. 3. The prompt vs delayed energy distribution of one AmBe sample (ACU-B $z=0 \mathrm{~m}$ ). The selected ground state (GS) and excited state (ES) are indicated. Negative bin content is due to background subtraction.

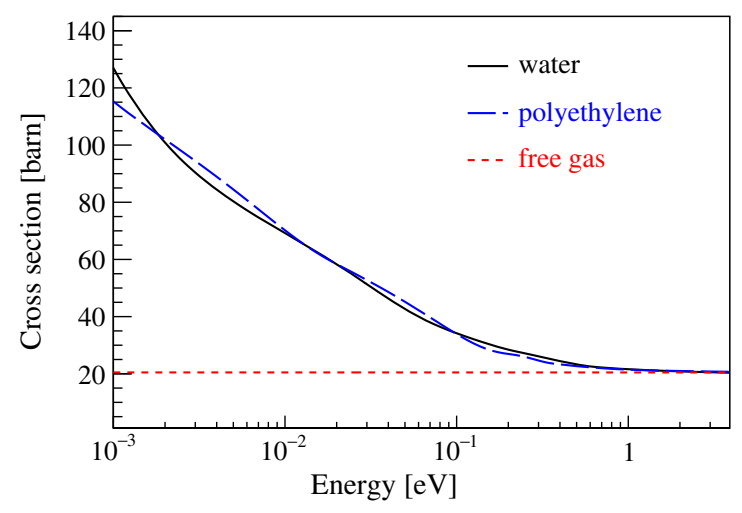

FIG. 4. Total scattering cross section as a function of neutron kinetic energy for three models. The data are extracted from Geant4 simulation.

\section{B. Neutron and gamma modeling in simulation}

The neutron calibration data were compared to the model predictions obtained using the Geant4-based [38] (v4.9.2) Daya Bay Monte Carlo (MC) simulation framework NuWa $[3,30]$ with improvements to the calibration pipe geometry, detector energy response, and neutron transport modeling [39]. These modifications improve the agreement between the neutron calibration data [32] and simulation.

Neutrons lose energy through various scattering processes before capture on a nucleus. There are no scattering models in Geant4 for the Daya Bay scintillator (average hydrogen-to-carbon ratio $\mathrm{CH}_{1.61 \sim 1.64}$ ) or acrylic $\left(\mathrm{C}_{5} \mathrm{O}_{2} \mathrm{H}_{8}\right)$. Above $4 \mathrm{eV}$, a generic Geant 4 model, "G4NeutronHPElastic" can be selected for neutron simulation. Below $4 \mathrm{eV}$, three possible options from the Geant 4 data libraries are an elastic scattering model without molecular bonds ("free gas"), a water model $\left(\mathrm{H}_{2} \mathrm{O}\right)$, and a polyethylene model $\left(\mathrm{CH}_{2}\right.$, "poly") [38]. The latter two models are built based on the ENDF database [40] and are quite different from the free gas model. The total scattering cross section as a function of neutron kinetic energy for the three models is shown in Fig. 4. To approximate the Daya Bay (scintillator, acrylic) material pair, five combinations (Table II) of models were studied, including (a) (water, free gas) (b) (water, poly), (c) (poly, poly), (d) (poly, free gas), and (e) (free gas, free gas).

For the neutron capture gamma energy and multiplicity distributions, four different models (Table II) were selected, including (1) a native Geant 4 model, (2) a Geant4 model with the photon evaporation process, (3) a model based on the Nuclear Data Sheets by L. Groshev et al. [41], and (4) a model based on the measured single gamma distribution of nGd capture at Caltech [29]. The energy spectra of the deexcitation gammas of gadolinium-155 and 157 are shown in Figs. 5(a) and 5(b), respectively, for these models. The gamma model-3 has the hardest gamma spectra.

The 20 available combinations provided by the five neutron scattering model combinations $(\mathrm{a}-\mathrm{e})$ and the four 


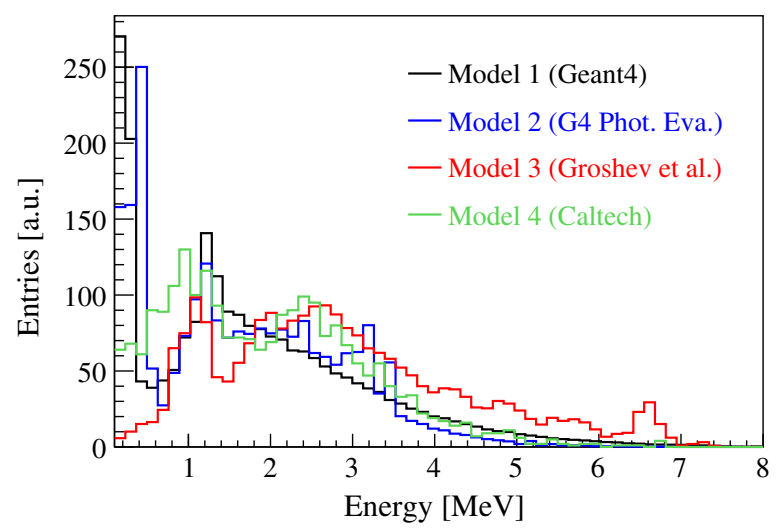

(a) ${ }^{155} \mathrm{Gd}$

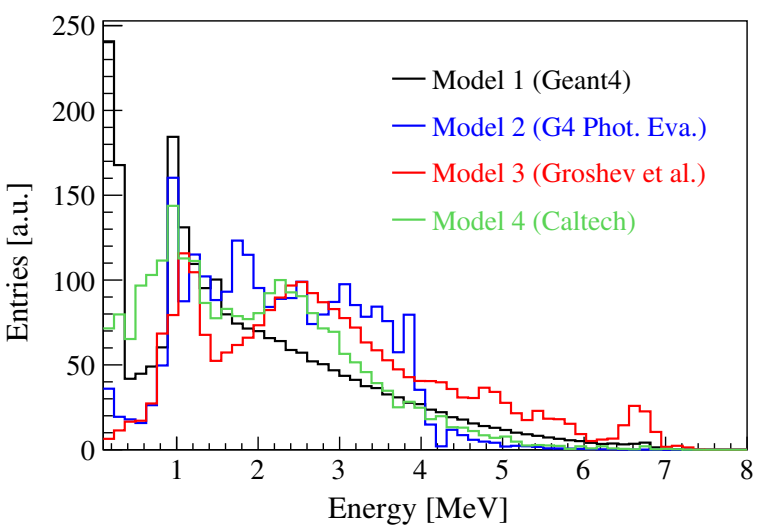

(b) ${ }^{157} \mathrm{Gd}$

FIG. 5. Energy distribution of the deexcitation gammas of ${ }^{155} \mathrm{Gd}$ (a) and ${ }^{157} \mathrm{Gd}$ (b) for the four models as indicated in the legend.

gamma models (1-4) are used to estimate $\varepsilon_{n}$. Model a-1 was used in the previous analyses $[29,32]$.

\section{Data and simulation comparison}

For each calibration SLP, a ratio $F$ is calculated.

$$
F=\frac{N([6,12] \mathrm{MeV})}{N([1.5,12] \mathrm{MeV})},
$$

where $N([6,12] \mathrm{MeV})$ and $N([1.5,12] \mathrm{MeV})$ are the numbers of events with reconstructed delayed energy in the range of $[6,12] \mathrm{MeV}$ and $[1.5,12] \mathrm{MeV}$, respectively. $1.5 \mathrm{MeV}$ is chosen to include the hydrogen capture peak. $F$ is very sensitive to the relative strength of $\mathrm{H}$ vs Gd capture peaks and the containment of the $8 \mathrm{MeV}$ of gamma energy from $n G d$ capture, and therefore provides a crucial benchmark for the neutron and gamma simulation models. For the 59 calibration SLPs, a $\chi^{2}$ is constructed to measure the overall difference between data and MC predictions,

$$
\chi^{2}=\left(F_{\text {data }}-F_{\mathrm{MC}}\right)^{T} \cdot V^{-1} \cdot\left(F_{\text {data }}-F_{\mathrm{MC}}\right),
$$

where $\left(F_{\text {data }}-F_{\mathrm{MC}}\right)$ is a vector with 59 elements of the difference of $F$ between the data and MC, and $V$ is the covariance matrix $(59 \times 59)$. For $47 \mathrm{SLPs}, \mathrm{V}$ is diagonal and the uncertainty is determined by statistics only. Near the GdLS boundary, where $F$ changes rapidly with source position for $12 \mathrm{SLPs}$, the $F_{\text {data }}-F_{\mathrm{MC}}$ are partially correlated due to the common source $z$ location uncertainty of $7 \mathrm{~mm}$ (Sec. III A). The $\chi^{2}$ and $\varepsilon_{n}$ values for all models are shown in Table II. The eight combinations with either neutron model-e or gamma model-3 are discrepant $\left(\chi^{2}>300\right)$, and therefore are excluded. The remaining twelve $(4 \times 3)$ models with $\chi^{2}$ in the range of 52.1-156 are called group $\mathrm{A}$. The best model with minimum $\chi^{2}$ is model b-1. In Fig. 6, the delayed energy spectra at two boundary calibration locations from data are compared to models b-1 and e- 1 , where model b- 1 shows a better agreement with data for $F$.

The data and best MC $F$ values and their differences are shown explicitly in Figure 7 for all sources and locations. The systematic variations among the twelve models in group A are overlaid, where the full spread among them, maximum minus minimum, are plotted as the gray bars. The variation in $F$ from $1 \%$ to $85 \%$ for the 59 data points is due to the differences in the local geometry and neutron kinetic energy and is well reproduced by simulation. For most SLPs, the best MC model, b-1, reaches an agreement with data at the subpercent level, and the residual difference is mostly smaller than the model spread. The 59 values of $\left(F_{\mathrm{MC}}-F_{\text {data }}\right) / \sigma$ for Model b-1 are projected in Fig. 8, where the $\sigma$ is the combined uncertainty from data and $\mathrm{MC}$

TABLE II. Summary of five neutron scattering model combinations and four gamma models, including the efficiency for detecting inverse beta decay neutrons and the $\chi^{2}$ with 59 calibration source-location points. See text for details.

\begin{tabular}{lcccc}
\hline \hline$\varepsilon_{n}, \chi^{2}$ & 1. Geant4 native & 2. Geant4 Phot. Eva. & 3. Nuclear Data Sheets & 4. Caltech \\
\hline a. water, free gas & $82.23 \%, 76.0$ & $82.35 \%, 86.4$ & $80.56 \%, 316$ & $82.55 \%, 156$ \\
b. water, poly & $81.75 \%, 52.1$ & $81.93 \%, 85.1$ & $80.42 \%, 350$ & $82.43 \%, 119$ \\
c. poly, poly & $81.61 \%, 56.6$ & $82.00 \%, 63.9$ & $79.96 \%, 389$ & $82.00 \%, 96.9$ \\
d. poly, free gas & $82.01 \%, 57.7$ & $82.28 \%, 79.9$ & $80.28 \%, 371$ & $82.36 \%, 115$ \\
e. free gas, free gas & $84.76 \%, 1183$ & $84.65 \%, 1273$ & $82.70 \%, 576$ & $85.37 \%, 1569$ \\
\hline \hline
\end{tabular}




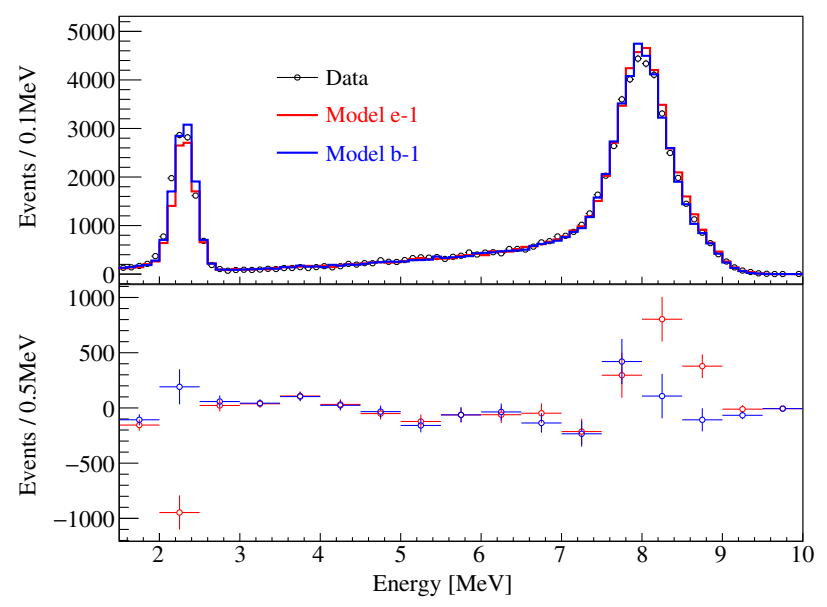

(a)AmBe excited state at $r=1.35 \mathrm{~m}$ and $z=-1.35 \mathrm{~m}$

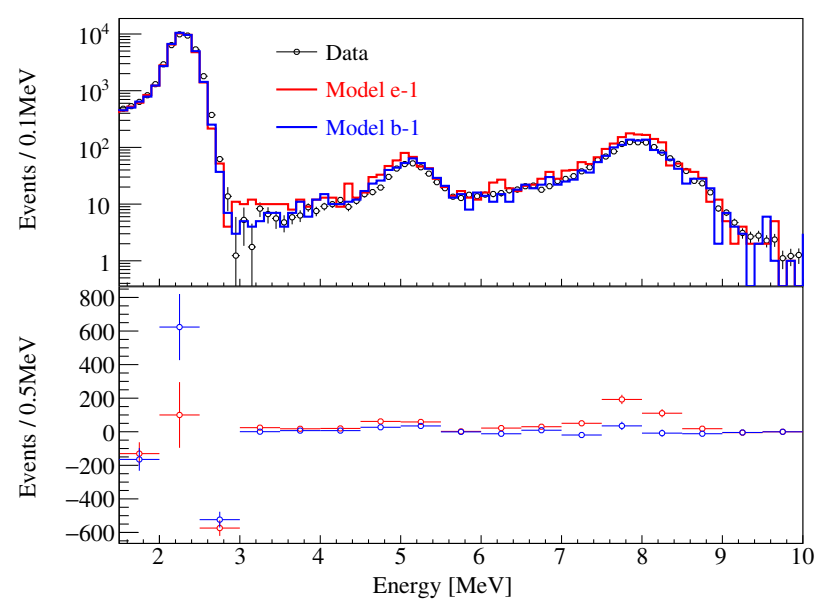

(b)AmC ground state at $r=1.77 \mathrm{~m}$ and $z=0 \mathrm{~m}$

FIG. 6. Delayed energy spectra of neutron sources at two calibration locations for data and simulation and the corresponding residual plots (MC-data). Two MC models, b-1 (best fit) and e-1 (rejected), are overlaid. Normalization is determined using the integral between 1.5 and $12 \mathrm{MeV}$. The difference of relative gadolinium/hydrogen capture ratio between subfigure (a) and (b) is due to the relative position to the GdLS volume. In (b), a weak signal of neutron capture on carbon can be seen. In (b), a mismatch of the energy scales of data and $\mathrm{MC}$ at $\mathrm{nH}$ peak is observed, but our selection cut efficiency is not sensitive to the difference.

for each SLP. The distribution is fitted with a Gaussian function. The fitted Gaussian mean and standard deviation are $0.19 \pm 0.18$ and $1.08 \pm 0.21$, respectively, in agreement with a unit normal distribution. The minimal and maximal means given by the 12 models of group A are -0.096 and 1.15 , respectively. The best model and data show a good global and local agreement, and their discrepancy is covered by the variance of the group A models.

Another quantity is also constructed, $F^{\prime}=$ $N([3,4.5] \mathrm{MeV}) / N([3,12] \mathrm{MeV})$, which is also sensitive to the gamma model and energy leakage. The same

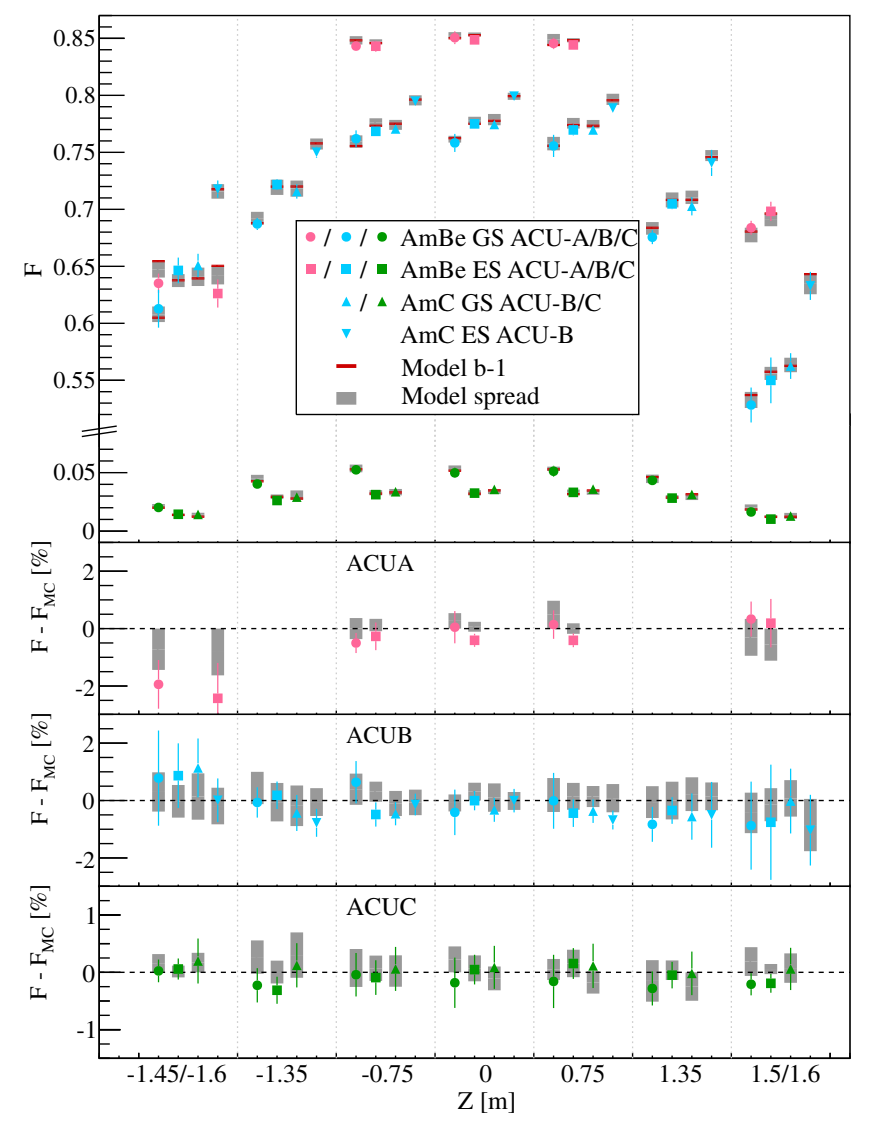

FIG. 7. Upper panel: The measured and best MC model (b-1) values of $F$ for $\mathrm{AmC}$ and $\mathrm{AmBe}$ (ground and excited states) neutrons at three calibration axes and different $z$ positions. The model spread of $F$, maximum minus minimum, for each sourcelocation point are also shown. The results at $z=-1.45 \mathrm{~m}$ and $-1.6 \mathrm{~m}$ are plotted together as are $1.5 \mathrm{~m}$ and $1.6 \mathrm{~m}$. Lower three panels: The difference of data and best MC in $F$ along the three vertical calibration axes, with the data and $\mathrm{MC}$ uncertainties combined. The gray bars indicate the spread of the twelve MC models in group A relative to the best model.

data-model comparison procedure confirms that gamma model 3 should be rejected and that gamma model 1 is reasonable.

To further investigate potential effects due to the discreteness of the calibration sources and the energy difference between neutron sources and IBD neutrons, we exploited a large sample of IBDs from data as a special SLP to be compared to the model prediction. Due to the resolution in position reconstruction, selection of pure GdLS IBDs is impossible. Instead, a GdLS + LS IBD sample from all four near site ADs were selected using cuts identical to those used on the neutron calibration data (Sec. III A), except that the prompt energy cut was adjusted to be greater than $3.5 \mathrm{MeV}$ to suppress accidental background. About 2 million GdLS + LS IBD events in total were selected. The measured ratio $F$ is consistent $\mathrm{AD}$ to $\mathrm{AD}$ with an average of $47.1 \% \pm 0.1 \%$. The ratio from 
model b- 1 is $47.0 \%$, and the full model spread is from $46.7 \%$ to $47.5 \%$.

\section{Neutron detection efficiency determination}

Each model can give a prediction on $\varepsilon_{n}$ for IBDs (see Table II). For the twelve models in group $\mathrm{A}, \varepsilon_{n}$ ranges from $81.61 \%$ (model c-1) to $82.55 \%$ (model a-4), and that from model b- 1 is $81.75 \%$ with a statistical uncertainty of $0.11 \%$. However, at some SLPs, small residual differences remain between the data and the best MC, as shown in Fig. 7 and Fig. 8. Instead of taking the prediction of the best $\mathrm{MC}$ as is, to have the most accurate central value for reactor neutrino flux, a correction is estimated.

One can translate the data and MC difference in $F$ to a correction to $\varepsilon_{n}$, since the two are intrinsically correlated (linear to the lowest order) through the neutron and gamma models mentioned above. In mathematical form, for the $i$ th SLP, we have

$$
\varepsilon_{n}=c_{i} \cdot\left(F_{\text {data }, i}-F_{\mathrm{MC}, \text { best }, i}\right)+\varepsilon_{\mathrm{MC}, \text { best }},
$$

where $\varepsilon_{\mathrm{MC} \text {,best }}$ is the neutron detection efficiency given by the best MC model. $c_{i}$ characterizes the linear correlation between $F_{i}$ and $\varepsilon_{n}$, and can be estimated through a linear regression (fit) using predicted values of $\varepsilon_{n}$ and $F_{i}$ from all 20 MC models. This procedure is illustrated in Fig. 9 for three representative SLPs. The fraction of accepted events, $F$, should be linearly proportional to the efficiency, $\varepsilon_{n}$, for variations in the neutron cross section as well as the gamma emission model. This relationship holds even for models that do not agree particularly well with our measurements, thus we utilize the full range of model space to estimate the correction to the neutron detection efficiency. The uncertainties in both axes are taken into account to perform the linear regression.

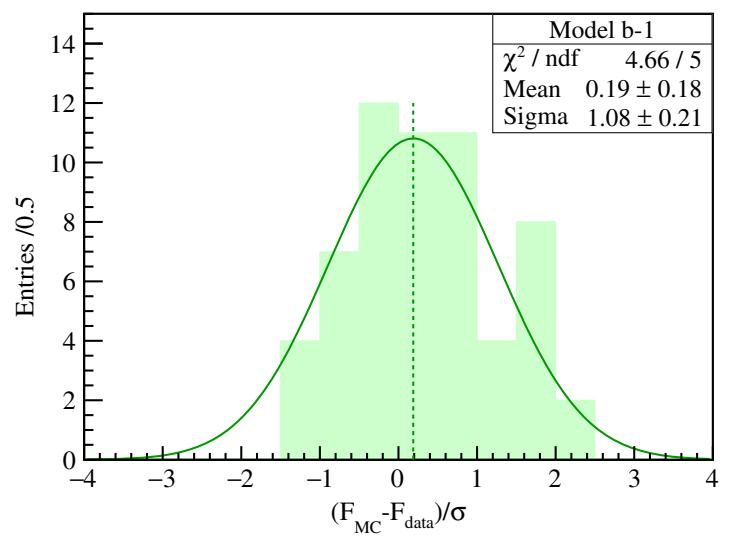

FIG. 8. The projection of $\left(F_{\mathrm{MC}}-F_{\text {data }}\right) / \sigma$ of the 59 SLPs for the best model b-1. The distribution is fitted with a Gaussian function. The fit result, as shown in top right panel, is consistent with a unit normal distribution, and the fitted mean is presented by the dashed line.

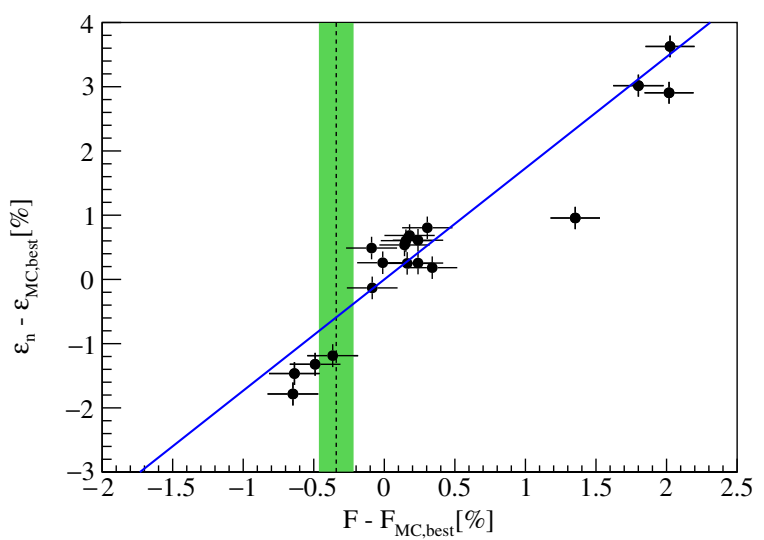

(a)AmBe excited state at $r=0 \mathrm{~m}$ and $z=-0.75 \mathrm{~m}$

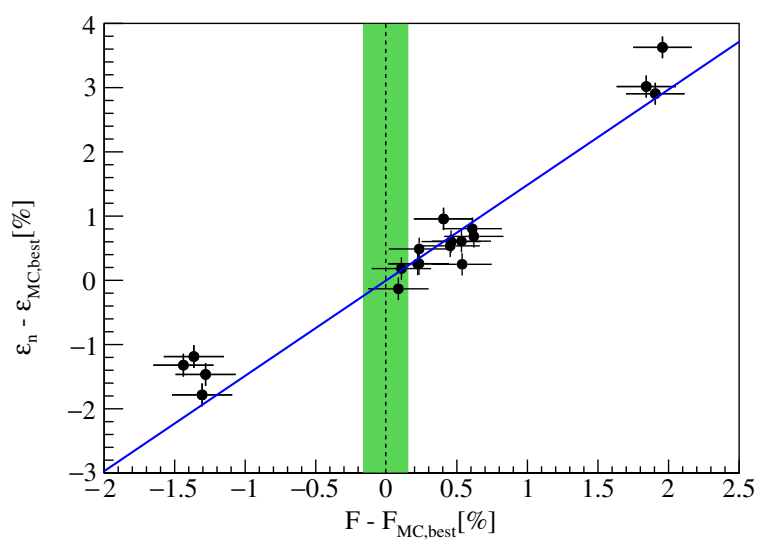

(b)AmBe excited state at $r=1.35 \mathrm{~m}$ and $z=0 \mathrm{~m}$

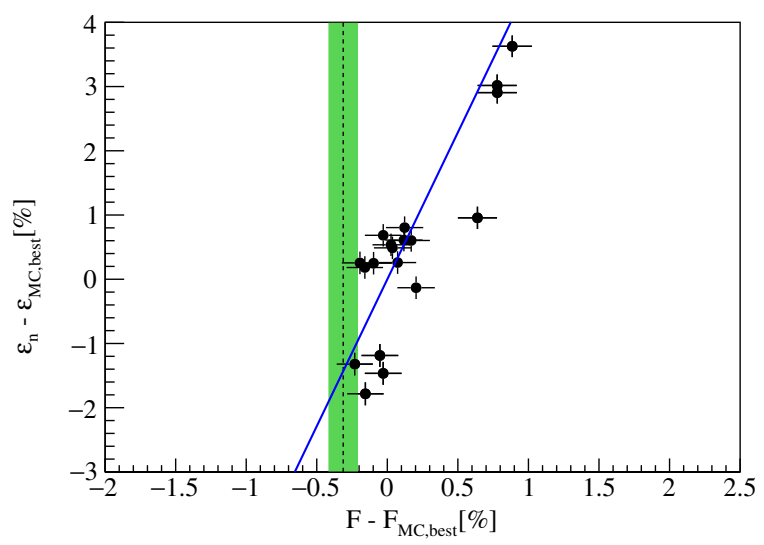

(c)AmBe excited state at $r=1.77 \mathrm{~m}$ and $z=-1.35 \mathrm{~m}$

FIG. 9. $\quad F-F_{\mathrm{MC}, \text { best }}$ vs $\varepsilon_{n}-\varepsilon_{\mathrm{MC} \text {,best }}$ for the $20 \mathrm{MC}$ models at three example SLPs. The vertical dashed line and the shaded band indicates the mean and uncertainty of measured $F$ with data. The vertical intercept of the linear fit passing through the best $\mathrm{MC}$ model (solid blue line) with the vertical dashed line gives the corrected value of $\varepsilon_{n}$.

Given the $c_{i}$ and $F_{\text {data }, i}$ at each SLP, a correction to $\epsilon_{n}$ can be computed using Eq. (6). The final average correction is obtained by making a weighted average of those from the 59 SLPs using the combined uncertainty from $c_{i}, F_{\text {data, } i}$, 
and $F_{\mathrm{MC}, i}$. The result is $-0.27 \%$ with a standard deviation of $0.49 \%$. Weighting the SLPs by the IBD fraction of the nearby volume produces consistent results. We also note that standard deviation is consistent with the half range of $\epsilon_{n}$ predicted by the group A models, $0.47 \%$ (Table II). Aside from the model uncertainties, other systematic effects (e.g., gadolinium abundance, source geometry, absolute energy scale, and material density variations) have been studied in the MC and found to be negligible. Based on the above, the final IBD neutron efficiency after the correction is $\varepsilon_{n}=((81.75-0.27) \pm 0.12$ (stat) \pm 0.47 (syst) $) \%=(81.48 \pm 0.60) \%$, in which the statistical and systematical uncertainties are combined linearly to capture potential correlations.

\section{ANTINEUTRINO YIELD AND COMPARISON WITH PREDICTION}

Using the new neutron detection efficiency $\varepsilon_{n}$ and Eq. (2), the IBD detection efficiency $\varepsilon_{\mathrm{IBD}}$ is $(80.25 \pm 0.61) \%$. Using the procedure as in Eq. (1), the mean IBD reaction yield per nuclear fission is

$$
\sigma_{f}=(5.91 \pm 0.09) \times 10^{-43} \mathrm{~cm}^{2} / \text { fission, }
$$

where the major uncertainties (Table I) are from the target proton fraction $0.92 \%$ (relative uncertainty), dominated by the hydrogen-to-carbon ratio due to instrumental uncertainty in the combustion measurements, and reactor-related uncertainty $0.90 \%$ (relative uncertainty) due to reactor power and fission fractions.

The ratio of the yield to the prediction of the HuberMueller (or ILL-Vogel) reactor model can be calculated. The effective fission fractions for four fission isotopes are defined as

$$
f_{\text {iso }}=\sum_{d=1}^{4} \sum_{r=1}^{6} \frac{N_{d}^{P} P_{s u r}^{r d} N_{r}^{f, \text { iso }}}{L_{r d}^{2}} / \sum_{d=1}^{4} \sum_{r=1}^{6} \frac{N_{d}^{P} P_{s u r}^{r d} N_{r}^{f}}{L_{r d}^{2}},
$$

where iso refers to one of the four major fission isotopes, i.e., ${ }^{235} \mathrm{U},{ }^{238} \mathrm{U},{ }^{239} \mathrm{Pu}$, and ${ }^{241} \mathrm{Pu}, N_{r}^{f \text {,iso }}$ is the predicted number of fissions contributed by the $i s o^{\text {th }}$ isotope in the $r$ th reactor core, and other symbols are defined in Eq. (1). In the analyzed data, the effective fission fractions for the four fission isotopes $\left({ }^{235} \mathrm{U},{ }^{238} \mathrm{U},{ }^{239} \mathrm{Pu}\right.$, and ${ }^{241} \mathrm{Pu}$ ) are determined to be $(0.564,0.076,0.304$, and 0.056$)$, respectively. The predicted IBD yield is the sum due to all four isotopes, including corrections due to nonequilibrium effects,

$\sigma_{f}=\sum_{\text {iso }=1}^{4} f_{\text {iso }} \int\left(S_{\text {iso }}\left(E_{\nu}\right)+k_{\text {iso }}^{\mathrm{NE}}\left(E_{\nu}\right)\right) \sigma_{\mathrm{IBD}}\left(E_{\nu}\right) \mathrm{d} E_{\nu}$,

in which $S_{\text {iso }}\left(E_{\nu}\right)$ is the predicted antineutrino spectrum for each isotope given by Huber-Mueller or ILL-Vogel model,
$\sigma_{\mathrm{IBD}}\left(E_{\nu}\right)$ is the IBD cross section, and $k_{\mathrm{iso}}^{\mathrm{NE}}\left(E_{\nu}\right)$ corrects for the nonequilibrium long-lived isotopes. The calculation integrates over neutrino energy $E_{\nu}$ and the nonequilibrium effect contributes $+0.6 \%$ [29]. The ratio between the measured to predicted reactor antineutrino yield $R$ is $0.952 \pm$ $0.014 \pm 0.023$ (Huber-Mueller) and $1.001 \pm 0.015 \pm$ 0.027 (ILL-Vogel), where the first uncertainty is experimental and the second is due to the reactor models themselves. A breakdown of the experimental uncertainties can be seen in Table I (see also Ref. [29]). The uncertainties from power, spent fuel, and nonequilibrium are treated to be uncorrelated among different reactor cores in the oscillation analysis [32], and those from fission fraction, IBD cross section, and energy/fission are treated to be correlated. They are conservatively treated as fully correlated in this analysis, and the total reactor-related uncertainty is $0.9 \%$. The total experimental uncertainty has been reduced to $1.5 \%$, which is a relative $29 \%$ improvement on our previous study. The new flux measurement is consistent with the ILL-Vogel model, but differs by 1.8 standard deviations with respect to the Huber-Mueller model, with the uncertainty now dominated by the theoretical uncertainty.

With the new result, a comparison with the other measurements $([29,42])$ is updated using the same method presented in Ref. [29]. A summary figure is shown in Fig. 10. The Daya Bay new result on $R$ is consistent with the world data. The new world average of $R$ is $0.945 \pm$ 0.007 (exp) \pm 0.023 (model) with respect to the HuberMueller model. This more precise measurement further indicates that the origin of RAA is unlikely to be due to detector effects.

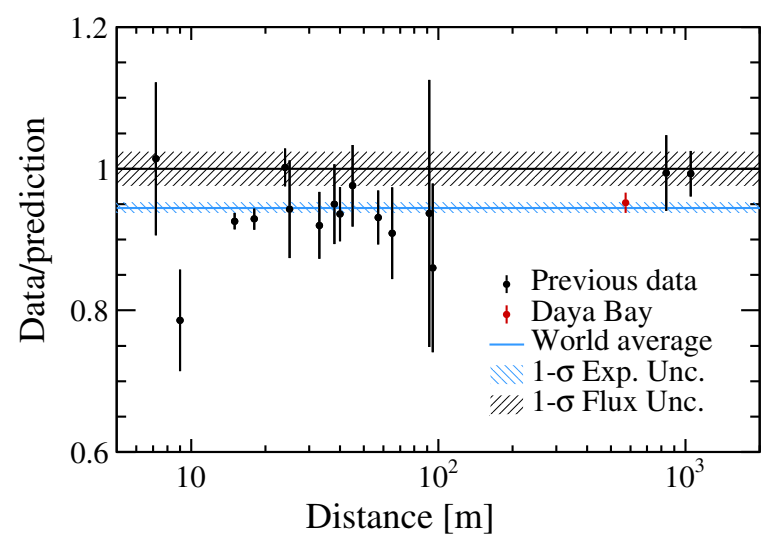

FIG. 10. The ratio of measured reactor antineutrino yield to the Huber + Mueller theoretical prediction as a function of the distance from the reactor to detector. Each ratio is corrected for the effect of neutrino oscillation. The blue shaded region represents the global average and its $1 \sigma$ uncertainty. The $2.4 \%$ model uncertainty is shown as a band around unity. The measurements at the same baseline are combined together for clarity. The Daya Bay measurement is shown at the flux weighted baseline $(578 \mathrm{~m})$ of the two near halls. 


\section{SUMMARY}

In summary, an improved antineutrino flux measurement is reported at Daya Bay with a 1230-day data set. The precision of the measured mean IBD yield is improved by $29 \%$ with a significantly improved neutron detection efficiency estimation. The new reactor antineutrino flux is $\sigma_{f}=(5.91 \pm 0.09) \times 10^{-43} \mathrm{~cm}^{2} /$ fission. The ratio with respect to predicted reactor antineutrino yield $R$ is $0.952 \pm$ $0.014 \pm 0.023$ (Huber-Mueller) and $1.001 \pm 0.015 \pm$ 0.027 (ILL-Vogel) [43], where the first uncertainty is experimental and the second is due to the reactor models. This yield measurement is consistent with the world data, and further confirms the discrepancy between the world reactor antineutrino flux and the Huber-Mueller model.

\section{ACKNOWLEDGMENTS}

The Daya Bay Experiment is supported in part by the Ministry of Science and Technology of China, the U.S.
Department of Energy, the Chinese Academy of Sciences, the CAS Center for Excellence in Particle Physics, the National Natural Science Foundation of China, the Guangdong provincial government, the Shenzhen municipal government, the China General Nuclear Power Group, the Research Grants Council of the Hong Kong Special Administrative Region of China, the Ministry of Education in Taiwan, the U.S. National Science Foundation, the Ministry of Education, Youth, and Sports of the Czech Republic, the Charles University Research Centre UNCE, the Joint Institute of Nuclear Research in Dubna, Russia, the NSFC-RFBR joint research program, the National Commission of Scientific and Technological Research of Chile, We acknowledge Yellow River Engineering Consulting Co., Ltd., and China Railway 15th Bureau Group Co., Ltd., for building the underground laboratory. We are grateful for the ongoing cooperation from the China Guangdong Nuclear Power Group and China Light \& Power Company.
[1] C. L. Cowan, F. Reines, F. B. Harrison, H. W. Kruse, and A. D. McGuire, Science 124, 103 (1956).

[2] K. Eguchi et al. (KamLAND Collaboration), Phys. Rev. Lett. 90, 021802 (2003).

[3] F. P. An et al. (Daya Bay Collaboration), Phys. Rev. Lett. 108, 171803 (2012).

[4] J. K. Ahn et al. (RENO Collaboration), Phys. Rev. Lett. 108, 191802 (2012).

[5] B. Pontecorvo, Zh. Eksp. Teor. Fiz. 33, 549 (1957) [Sov. Phys. JETP 6, 429 (1957)].

[6] B. Pontecorvo, Zh. Eksp. Teor. Fiz. 53, 1717 (1967) [Sov. Phys. JETP 26, 984 (1968)].

[7] Z. Maki, M. Nakagawa, and S. Sakata, Prog. Theor. Phys. 28, 870 (1962).

[8] P. Huber, Phys. Rev. C 84, 024617 (2011).

[9] T. A. Mueller et al., Phys. Rev. C 83, 054615 (2011).

[10] G. Mention, M. Fechner, T. Lasserre, T. A. Mueller, D. Lhuillier, M. Cribier, and A. Letourneau, Phys. Rev. D 83, 073006 (2011).

[11] W. G. K. Schreckenbach, G. Colvin, and F. von Feilitzsch, Phys. Lett. 160B, 325 (1985).

[12] F. von Feilitzsch, A. Hahn, and K. Schreckenbach, Phys. Lett. 118B, 162 (1982).

[13] A. Hahn, K. Schreckenbach, W. Gelletly, F. von Feilitzsch, G. Colvin, and B. Krusche, Phys. Lett. B 218, 365 (1989).

[14] P. Vogel, G. K. Schenter, F. M. Mann, and R. E. Schenter, Phys. Rev. C 24, 1543 (1981).

[15] J. Kopp, M. Maltoni, and T. Schwetz, Phys. Rev. Lett. 107, 091801 (2011).

[16] J. Kopp, P. A. N. Machado, M. Maltoni, and T. Schwetz, J. High Energy Phys. 05 (2013) 050.

[17] C. Giunti, M. Laveder, Y. F. Li, and H. W. Long, Phys. Rev. D 88, 073008 (2013).
[18] C. Athanassopoulos et al. (LSND Collaboration), Phys. Rev. Lett. 81, 1774 (1998).

[19] A. Aguilar-Arevalo et al. (LSND Collaboration), Phys. Rev. D 64, 112007 (2001).

[20] A. A. Aguilar-Arevalo et al. (MiniBooNE Collaboration), Phys. Rev. Lett. 105, 181801 (2010).

[21] W. Hampel et al. (GALLEX Collaboration), Phys. Lett. B 420, 114 (1998).

[22] J. N. Abdurashitov et al. (SAGE Collaboration), Phys. Rev. C 80, 015807 (2009).

[23] C. Giunti, Phys. Rev. D 96, 033005 (2017).

[24] M. Dentler, 1. Hernández-Cabezudo, J. Kopp, M. Maltoni, and T. Schwetz, J. High Energy Phys. 11 (2017) 099.

[25] C. Giunti, X. P. Ji, M. Laveder, Y. F. Li, and B. R. Littlejohn, J. High Energy Phys. 10 (2017) 143.

[26] Y. Gebre, B. R. Littlejohn, and P. T. Surukuchi, Phys. Rev. D 97, 013003 (2018).

[27] F. P. An et al. (Daya Bay Collaboration), Phys. Rev. Lett. 118, 251801 (2017).

[28] F. P. An et al. (Daya Bay Collaboration), Phys. Rev. Lett. 116, 061801 (2016); 118, 099902(E) (2017).

[29] F. P. An et al. (Daya Bay Collaboration), Chin. Phys. C 41, 013002 (2017).

[30] F. P. An et al. (Daya Bay Collaboration), Nucl. Instrum. Methods Phys. Res., Sect. A 811, 133 (2016).

[31] F. P. An et al. (Daya Bay Collaboration), Phys. Rev. D 93, 072011 (2016).

[32] F. P. An et al. (Daya Bay Collaboration), Phys. Rev. D 95, 072006 (2017).

[33] P. Vogel and J.F. Beacom, Phys. Rev. D 60, 053003 (1999).

[34] K. A. Olive et al. (Particle Data Group), Chin. Phys. C 38, 090001 (2014). 
[35] X. B. Ma, W. L. Zhong, L. Z. Wang, Y. X. Chen, and J. Cao, Phys. Rev. C 88, 014605 (2013).

[36] J. Liu, R. Carr, D. A. Dwyer, W. Q. Gu, G. S. Li, R. D. McKeown, X. Qian, R. H. M. Tsang, F.F. Wu, and C. Zhang, Nucl. Instrum. Methods Phys. Res., Sect. A 797, 260 (2015).

[37] J. Liu et al., Nucl. Instrum. Methods Phys. Res., Sect. A 750, 19 (2014).

[38] S. Agostinelli et al. (GEANT4 Collaboration), Nucl. Instrum. Methods Phys. Res., Sect. A 506, 250 (2003).

[39] An error in neutron propagation was corrected. Details can be found in https://bugzilla-geant4.kek.jp/show_bug.cgi? $\mathrm{id}=1856$. We replaced the G4UHadronElasticProcess with G4HadronElasticProcess to correct the error, and the IBD selection efficiency increased by $0.3 \%$.

[40] M. B. Chadwick et al., Nucl. Data Sheets 112, 2887 (2011).

[41] L. Groshev et al., Nucl. Data Sheets, Sect. A 5, 1 (1968).

[42] G. Boireau et al. (Nucifer Collaboration), Phys. Rev. D 93, 112006 (2016).

[43] It is noteworthy that although the ILL-Vogel model agrees well with the data in total flux, its predicted energy spectrum (and that from the Huber-Mueller) is in strong tension with the experimental IBD antineutrino spectrum. 